\title{
Unmanned Surveying System using Automatic Moving Vehicle
}

\author{
Takahiro Kishishita \\ Kazuhiro Jo \\ Shinichi Sako \\ Shigetosi Koga
}

\author{
Technical Research Institute, Fujita Corporation \\ 74 Ohdana-cho Kouhoku-Ku, Yokohama, 223 Japan
}

\begin{abstract}
This paper describes a new automatic survey system developed for saving a shortage of workers in construction sites. The system consists of a three dimensional surveying system, a self-handling system and a personal computer, which are mounted on a fixed station and a moving station separately, being able to survey the geography without any operators. Some tests were carried out to investigate the performance both in laboratory and field. The results show that three dimensional coordinate of a target can be surveyed automatically and continuously with high accuracy, and that the system can be handled easily.
\end{abstract}

\section{INTRODUCTION}

The shortage of workers in construction site has been becoming a serious problem more and more in Japan, especially a lack of well-skilled worker is the most serious problem. To overcome these situations, many kinds of automatic constructing system have been developed. In a construction site, the surveying works are necessary for setting up a position of structures, a management of progressing work and a monitoring. The surveying work, however, is one of which needs well-skilled workers, therefore an automatic surveying system which can be handled easily and without any operator has been needed. A new automatic surveying system has been developed by the authors, aimed to be used mainly for a management of progressing work, for instance, a calculation of an area or a calculation of soil mass.

\section{SYSTEM OVERVIEW}

The present system consists of a three dimensional surveying system, a self-handling system and a personal computer which calculates a data and outputs results. Figure 1 and Figure 2 show photographs of this system and Figure 3 shows a block diagram.

\subsection{Surveying System}

The surveying is performed by using an optical communication device and an electro-optical distance meter. The electro-optical distance meter of the fixed station and the prism of the mobile station are keeping face-to-face each other by automatic tracking devices. Since the coordinate of the fixed station is known previously, a three dimensional coordinate of the target can be calculated through a distance, a horizontal angle and vertical angle which are obtained by the electro-optical distance meter. During the mobile station is moving, the system can survey a target continuously. The capacity of getting data is ten times a second. 


\section{2 Self-handling System}

This system consists of a main control unit, an operation control circuit, a start/stop switch of the engine, a forward/backward switch, a steering mechanism (4WS/parallel steering) and a braking device. Based on an information transmitted from the fixed station through the optical communication device, the vehicle is handled by personal computer mounted on the vehicle. The computer calculates the direction which the vehicle should be headed and moves the vehicle for a target. After arriving the target, the vehicle is turned toward the next target.

\subsection{Monitoring System of the Safety}

An ultrasonic sensor and a touch sensor are used to detect an obstacle around the vehicle. The detectors of pitching and rolling angle detect the balance of the vehicle. Data detected from these sensors is transmitted to the main control unit of the mobile station in order to avoid an obstacle or to do emergency stop as required.

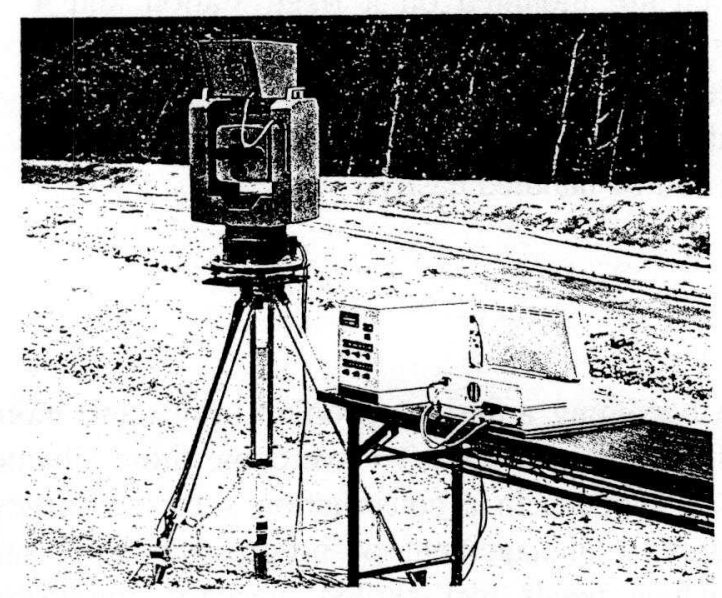

Figure 1 Photograph of fixed station

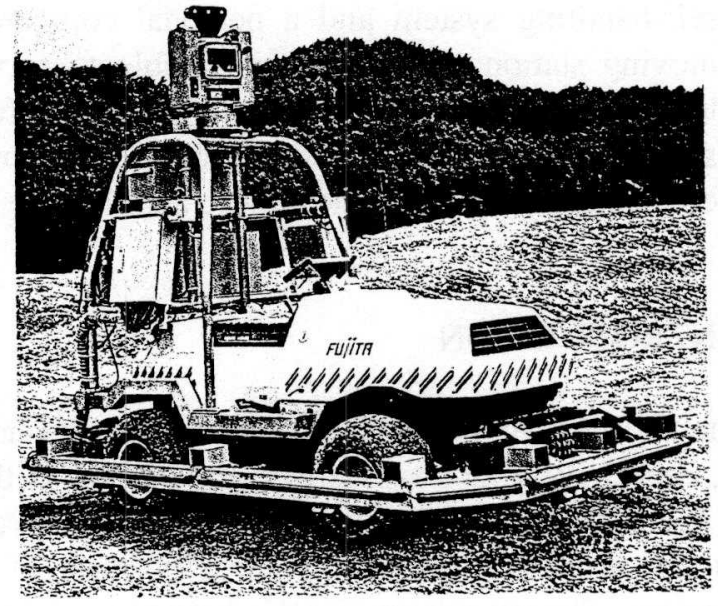

Figure 2 Photograph of mobile station

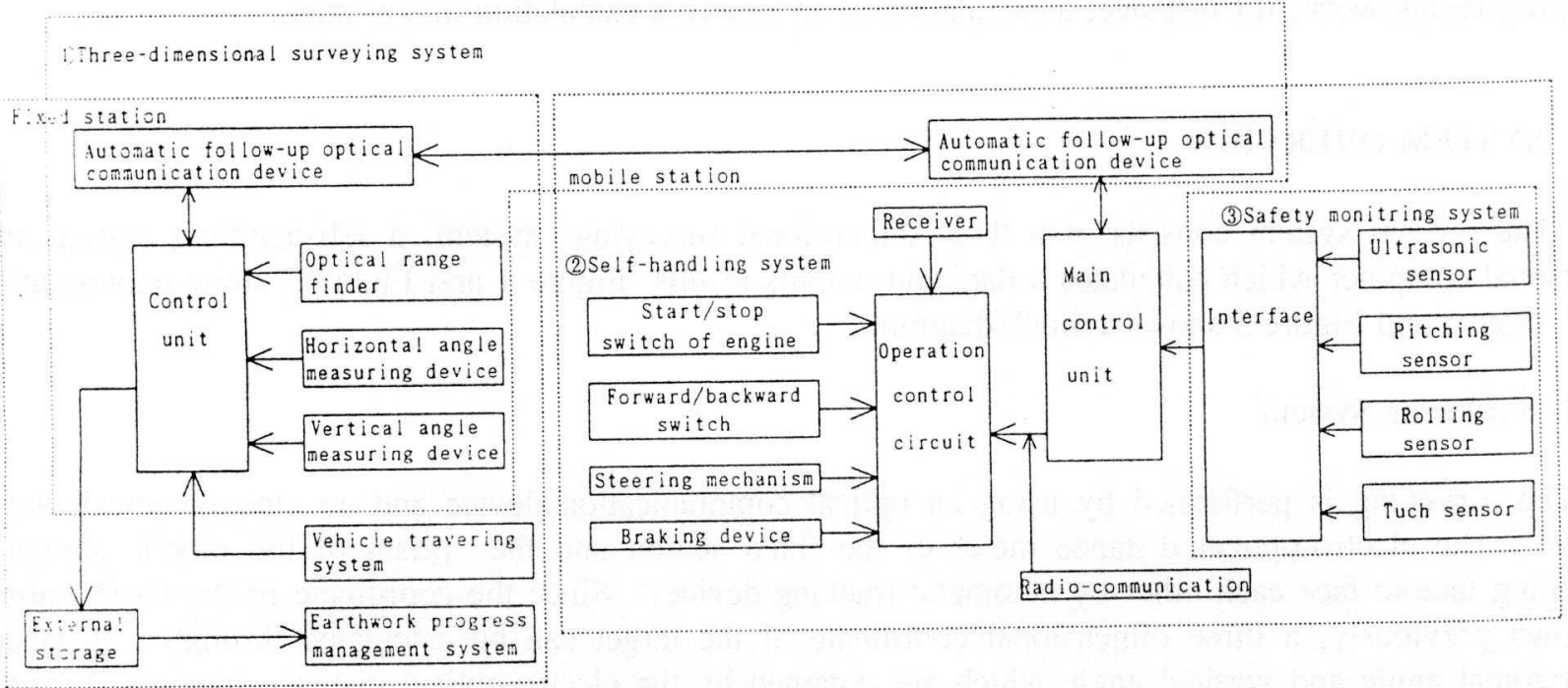

Figure 3 Block diagram of unnmanned survey system 


\subsection{Flow Chart of Surveying System}

The operational flow of the system is shown in Figure 4. Some key points are following.

1) A range of the surveying work, a coordinate of the fixed station and turning points of the route are inputted into the personal computer of the fixed station previously.

2) The fixed station transmitted an information to the mobile station, handling the operation of surveying.

3) The surveying vehicle autonomously moves based on the information of the surveying path and turning points stored in the microcomputer, being a target. The fixed station calculates the position of the target during the movement continuously.

4) After the surveying operation is completed, the personal computer of fixed station calculate and produces a cross section view and an over view for the management.

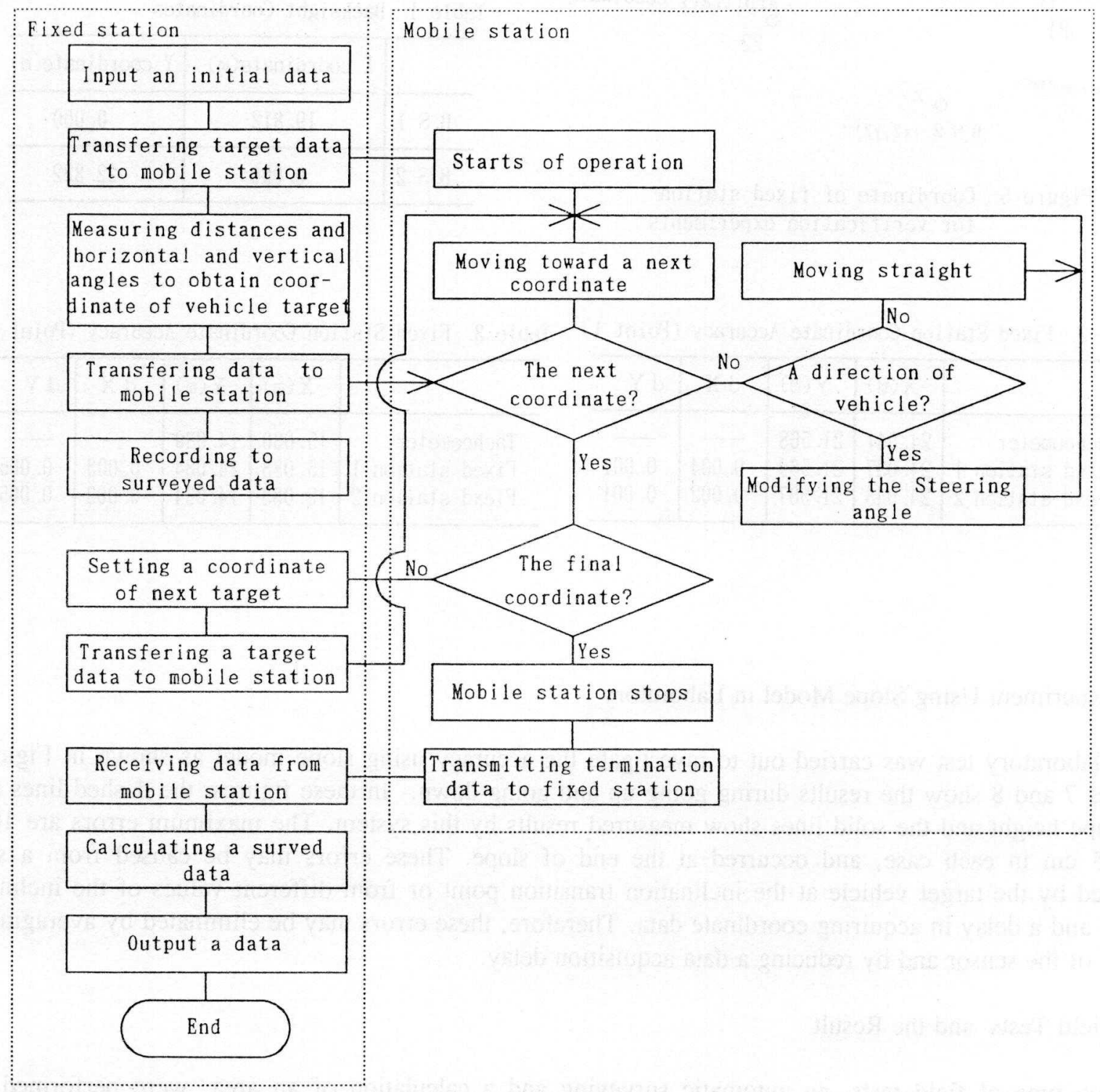

Figure 4 Flow chart of unnmanned survey svstem 


\section{EXPERIMENTS AND THE RESULTS}

Some tests were carried out both in laboratory and field, in order to make sure the performance and the accuracy of the system.

\subsection{Coordinate of Fixed Station.}

In this system, a coordinate of fixed station is calculated automatically based on coordinates of two back sight points which are known. Figure 5 shows the experimental set up and tests were performed at two points ( P1, P2). The points were measured by a tacheometer manually in order to estimate the accuracy. Tables 1 and table 2 show the results performed twice and the data obtained by the tacheometer. An error in any case is within $5 \mathrm{~mm}$.

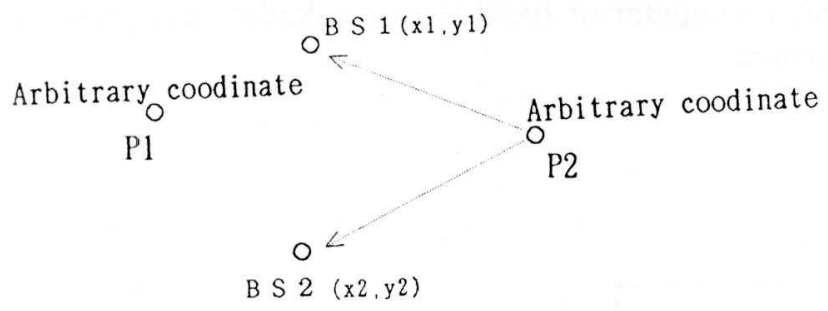

Figure 5 Coordinate of fixed station

Table 1. Backsight Coordinates

\begin{tabular}{c|c|c}
\hline & X coordinate $(\mathrm{m})$ & Y coordinate(m) \\
\hline B.S 1 & 19.812 & 0.000 \\
\hline B.S 2 & 0.000 & 22.822 \\
\hline
\end{tabular}
for verification experiments

Table 2. Fixed Station Coordinate Accuracy (Point 1)

\begin{tabular}{l|c|c|c|c}
\hline & $\mathrm{X}(\mathrm{m})$ & $\mathrm{Y}(\mathrm{m})$ & $\mathrm{d} \mathrm{X}$ & $\mathrm{d} \mathrm{Y}$ \\
\hline Tacheometer & 24.041 & 21.568 & - & - \\
Fixed station 1 & 24.037 & 21.569 & -0.004 & 0.001 \\
Fiexd station 2 & 24.043 & 21.567 & 0.002 & -0.001 \\
\hline
\end{tabular}

Table 3. Fixed Station Coordinate Accuracy (Point 2)

\begin{tabular}{l|c|c|c|c}
\hline & $\mathrm{X}(\mathrm{m})$ & $\mathrm{Y}(\mathrm{m})$ & $\mathrm{d} \mathrm{X}$ & $\mathrm{d} \mathrm{Y}$ \\
\hline Tacheometer & 15.030 & 14.089 & - & - \\
Fixed station 1 & 15.033 & 14.084 & 0.003 & -0.005 \\
Fiexd station 2 & 15.033 & 14.084 & 0.003 & -0.005 \\
\hline
\end{tabular}

\subsection{Experiment Using Slope Model in Laboratory}

A laboratory test was carried out to investigate the accuracy using slope model as shown in Figure 6. Figures 7 and 8 show the results during going up and going down. In these figures, the dashed lines show the slope height and the solid lines show measured results by this system. The maximum errors are $10 \mathrm{~cm}$ and $15 \mathrm{~cm}$ in each case, and occurred at the end of slope. These errors may be caused from a shock received by the target vehicle at the inclination transition point or from different values of the inclination sensor and a delay in acquiring coordinate data. Therefore, these errors may be eliminated by averaging the values of the sensor and by reducing a data acquisition delay.

\subsection{Field Tests and the Result}

Two type of field tests, an automatic surveying and a calculation of an area, were performed in a construction site. In the former test, an unmanned surveying operation was performed and the vehicle was operated by an operator to measure an area in latter test. 


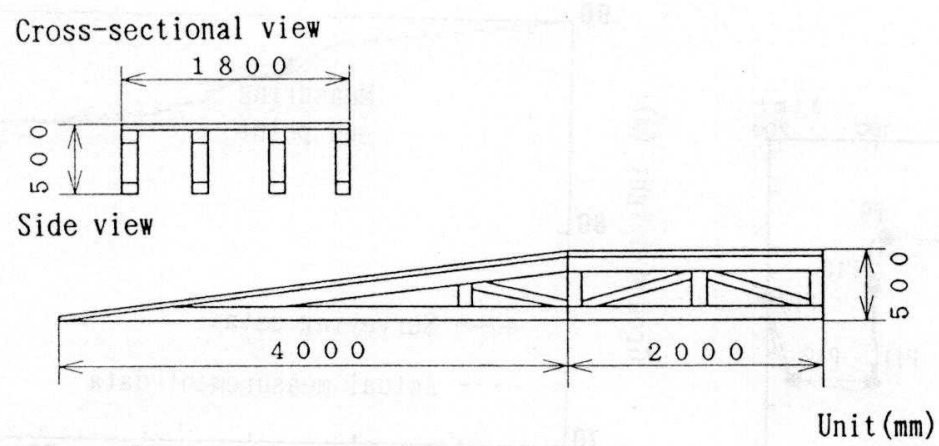

Figure 6 Cross section of experimental slope

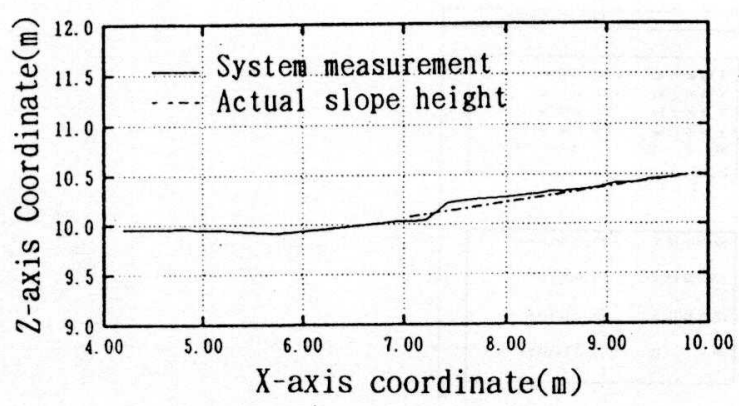

Figure 7 Results of experimental slope (Up)

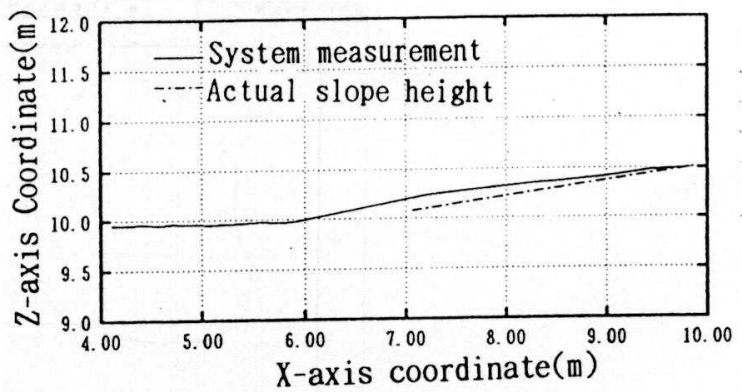

Figure 8 Results of experimental slope (Down)

\subsubsection{Automatic Surveying in the Field}

Figure 9 shows a plane view of the test path and the results obtained by automatic surveying system. The solid symbol indicates a turning point on the path and the solid line indicates the results. In order to investigate the accuracy, a measurement was also performed manually shown as dotted line. The comparison of two surveying results show that this automatic surveying system can get a data with high accuracy. For this experiment, the vehicle can turn a point within $60 \mathrm{~cm}$ of distance, though it is expected to be over $1 \mathrm{~m}$.

Figure 10 shows a cross section of $\mathrm{P} 1$ to $\mathrm{P} 2$ in Figure 9, and the symbol indicates a measuring range. The solid line and the dash line indicate the results obtained by this system and by manual measurement respectively. The maximum error is approximately $10 \mathrm{~cm}$ in high and may be caused by a same reason as described above.

\subsubsection{An Application to Measure an Area}

As an application of this system, a measurement of an area which is turfed was performed under being handled by an operator. Figure 11 shows the result calculated from the measured data. Table 4 shows a comparison between the area and the result from manual surveying. What the difference between two results is less than 0.7 percentage shows that this system has enough capability of surveying. And also this system takes 1 hour to get the result with a person, though the conventional method takes 12 hours with two persons for calculating the area. This means that this system is useful for saving a shortage of wellskilled worker in construction site. 


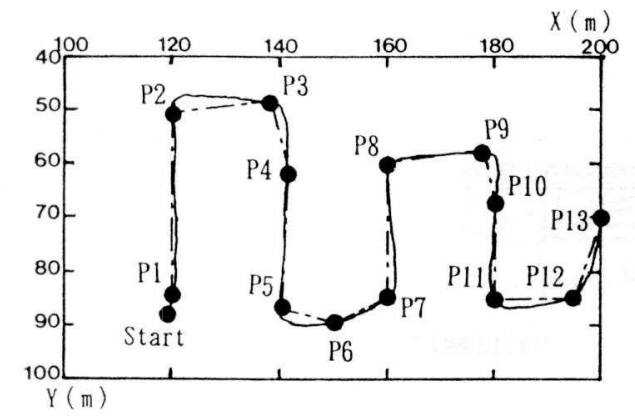

Figure 9 Heasuring path, transition point and surveying results

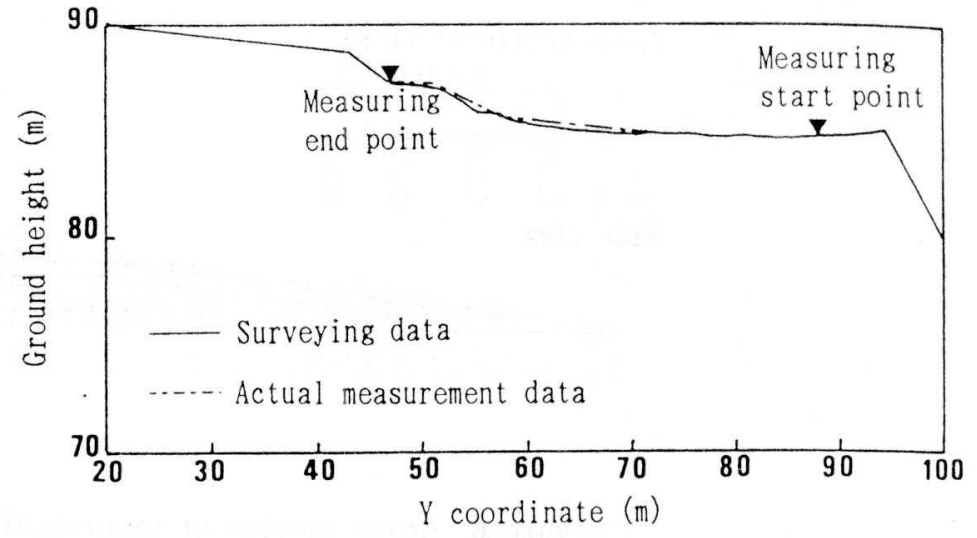

Figure 10 Cross section of surveying data

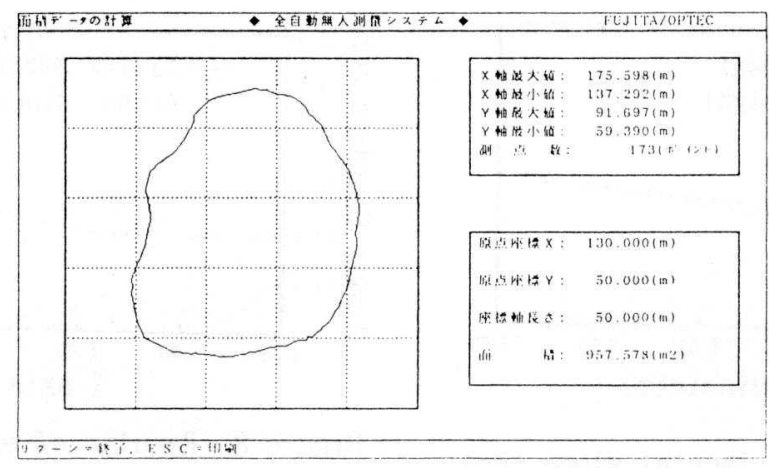

Figure 11 Results calculated by the present system

Table 4. Comparison between Results Caluculated with the Present System and by Plane-table Surveying

\begin{tabular}{l|c|c|l}
\hline & $\begin{array}{r}\text { Calculated } \\
\text { area (m2) }\end{array}$ & $\begin{array}{l}\text { Number of } \\
\text { personnel }\end{array}$ & $\begin{array}{l}\text { Time } \\
\text { required(H) }\end{array}$ \\
\hline Plane-table surveying & 964.010 & 2 & 12 \\
\hline Present system & 957.758 & 1 & 1 \\
\hline
\end{tabular}

\section{CONCLUSION}

The followings are advantage of this system.

(1) This system does not need a well-skilled worker for surveying works and saves a shortage of worker in construction site.

(2) Surveyed data can be produced continuously during the mobile station is moving. The frequency is a ten times a second.

(3) The vehicle can climb a slope of 30 degrees and can do excellent running in any rough area.

(4) The coordinate of fixed station can be obtained automatically by collimating two datum points after the station is installed. This makes it easy to turn the fixed station.

(5) The accuracy is much enough for a constructing management.

Further development of detectors of the balance of the vehicle and the handling technique are needed. 